\title{
Developments in the treatment of transfusion- dependent anemia in patients with myelodysplastic syndromes: epidemiology, etiology, genetics, and targeted therapies
}

\author{
This article was published in the following Dove Press journal: \\ Advances in Genomics and Genetics \\ 14 July 2014 \\ Number of times this article has been viewed
}

\author{
Azra Raza \\ Nicholas Iverson \\ Abdullah M Ali \\ The MDS Center, Columbia \\ University, New York, NY, USA
}

Correspondence: Azra Raza MDS Center, Columbia University Medical Center, Milstein Hospital Building, 6N-435, 177 Fort Washington Avenue, New York, NY 10032, USA

$\mathrm{Tel}+\mathrm{I} 212305829$ |

Fax +| $212305689 \mid$

Email azra.raza@columbia.edu

\begin{abstract}
Myelodysplastic syndromes are malignant hematopoietic stem cell disorders that present with variable cytopenias and predominantly affect the elderly. Treatment options are limited, with allogeneic transplant being the only potentially curative strategy. Recent mutational profiling studies have led to cataloguing of driver and passenger mutations most commonly affecting the epigenetic regulators and genes involved in RNA splicing. Despite improved understanding of the disease biology, these emerging molecular insights have not led to identification of novel therapeutic strategies. Although several drugs approved in the last decade improve the cytopenias, the relief is temporary, most likely due to the sequential activation of clones. Future advances depend upon identification of signaling pathways in dominant clones and targeting these with agents that might be known but need to be matched to suit the needs of individual patients in a longitudinal, dynamic fashion. Myelodysplastic syndromes are ideally suited for the development of such personalized medicine.
\end{abstract}

Keywords: cancer, epigenetics, iron, MDS, myelodysplasia, splicing

\section{Introduction}

Anemia is frequently encountered in the elderly, significantly impacting the quality of life through adversely affecting physical, emotional, social, and cognitive faculties. Myelodysplastic syndromes (MDS), as a cause of anemia due to primary bone marrow stem cell disorder, are acquiring increasing importance following the recognition that MDS has been under diagnosed in this age group and is more common than previously suspected. In fact, it is frequently the symptoms due to anemia that prompt patients to seek medical attention, unraveling a diagnosis of MDS. The incidence increases rather dramatically with advancing age, and MDS are currently established as the most common hematologic malignancy in the elderly: 75 per 100,000 in those aged $>65$ years, an increase from three to four per 100,000 in the general population. ${ }^{1}$ A European study reported that $87 \%$ of MDS patients presented with anemia at diagnosis, with $63 \%$ having hemoglobin levels of $<10 \mathrm{gm} / \mathrm{dL}$ and $29 \%$ being transfusion dependent; the incidence was $65 \%$ in patients with higher-risk MDS compared to $37 \%$ in the lower-risk group. ${ }^{2}$ According to the MDS Foundation sponsored survey, at least $70 \%$ of MDS patients are red blood cell (RBC)-transfusion dependent. ${ }^{3}$

Anemia worsens the comorbidities frequently present in the elderly and is associated with a wide spectrum of added complications. Management of chronic anemia and the resulting iron overload from frequent transfusions remain the key objectives in lower-risk 
MDS patients, where the disease itself may not be posing an imminent threat to life. Unfortunately, correction of chronic anemia with frequent transfusions is not without hazards. Frequent transfusions lead to development of alloantibodies and expose patients to allergic complications of skin rashes and anaphylaxis as well as iron overload. One study ${ }^{4}$ reported a higher incidence of infections in transfusion-dependent patients with MDS (81\% compared to 56\% in nontransfused patients). Several studies have identified serum ferritin levels to adversely affect survival, ${ }^{5}$ and the profundity of anemia is independently associated with overall and leukemia-free survival. ${ }^{6}$

Besides transfusion for the correction of anemia - a supportive care measure fraught with its own emotional, financial, and physical burdens - other treatments include the use of erythroid-stimulating agents (ESAs), immunomodulatory drugs (lenalidomide for $\operatorname{del}(5 q)$ MDS patients), hypomethylating drugs, and, where indicated, allogeneic stem cell transplant. In the following discussion, epidemiology, etiology, and prognosis of transfusion-dependent anemia in MDS will be reviewed with special emphasis on genetic factors and the development of current and targeted therapies.

\section{Epidemiology}

MDS was listed as a reportable malignant disease for the first time by The International Classification of Diseases for Oncology in 2000 and, in 2001, MDS became reportable to the Surveillance, Epidemiology, and End Results (SEER) Program, which consists of high-quality population-based cancer registries that are supported and sponsored by the National Cancer Institute. SEER data indicated that the risk of MDS increased with age, and the incidence was consistent with what was reported previously in other population-based studies that were conducted in Europe. ${ }^{7}$ The disease was most common in white individuals with a median age at diagnosis of 76 years, and the SEER incidence was three to four per 100,000 person-years. This may be an underestimate due to incomplete case registration and under-diagnosis. Nonetheless, approximately $86 \%$ of MDS cases were diagnosed in individuals aged $\geq 60$ years, with a significantly higher rate in men compared to women (4.5 versus 2.7 per 100,000 per year). In 2003, approximately 10,300 incident cases of MDS were diagnosed in the United States. Pooling data from several studies, there are an estimated 12,000 new MDS cases diagnosed per year in the US and 20,000 in Europe.

As far as prevalence is concerned, it has been calculated that 170,000 persons are living with MDS in the US based on The Third National Health and Nutrition Examination
Survey cross-sectional study, which showed that $11.0 \%$ of men and $10.2 \%$ of women were anemic, and that $5.8 \%$ of these anemic patients manifested features in the peripheral blood suggestive of MDS; however, this is likely to be an overestimate. ${ }^{8}$ The number of prevalent cases clearly depends upon survival, which is inversely related to the risk of transformation to leukemia. The reported overall 3-year survival is generally poor for MDS, with an overall rate of 35\%; men diagnosed at an older age have significantly worse survival. ${ }^{7}$ Longer survival is reported for patients in Asia as compared to Western countries. ${ }^{9}$ Ultimately, the overall survival numbers are misleading and of little value for individual patients because of the tremendous heterogeneity of the disease. With improved and more effective therapies, some of which improve survival significantly, the prevalence rates will also have to be revised.

\section{Etiology}

MDS is sometimes divided into primary (or de novo) disease and secondary disease (which is due to prior cytotoxic therapy, particularly alkylating agents, also referred to as therapy-related MDS). Approximately $10 \%$ of MDS patients belong to the latter group based on a history of chemotherapy, radiation therapy, or other chemical exposure, especially to benzene, ${ }^{10,11}$ and their prognosis is generally worse compared to the de novo cases because response to treatment is poor and incidence of transformation to acute myeloid leukemia (AML) is high. ${ }^{12}$ While $~ 40 \%$ of de novo cases present with cytogenetic abnormalities, 95\% of patients with therapyrelated MDS are diagnosed with abnormalities associated with higher-risk disease such as complex karyotypes, monosomy 5, or deletions of chromosome 7. Examination of lifestyle issues such as obesity, smoking, alcohol consumption, and occupational exposures to pesticides and alcohol have yielded equivocal results in large meta-analyses, and none of these have been established as definitely causing MDS. A meta-analysis of nine studies found that exposure to benzene, an aromatic hydrocarbon, increases the risk of AML by 3.2-fold. While few of these benzene studies reported upon MDS patients separately, higher than expected numbers of MDS cases were definitely recorded in some (Figure 1). ${ }^{12}$ The association of MDS with solvents is stronger for higher-risk patients with refractory anemia and excess blasts (RAEB) compared to RA or RA with ringed sideroblasts (RARS). In terms of genetics, the detoxifying glutathione-S-transferase genes (GSTT1) and an erythropoietin (EPO) promoter gene variant (rs 1617640) associated with decreased EPO expression have been related to increased incidence of MDS. ${ }^{13}$ 


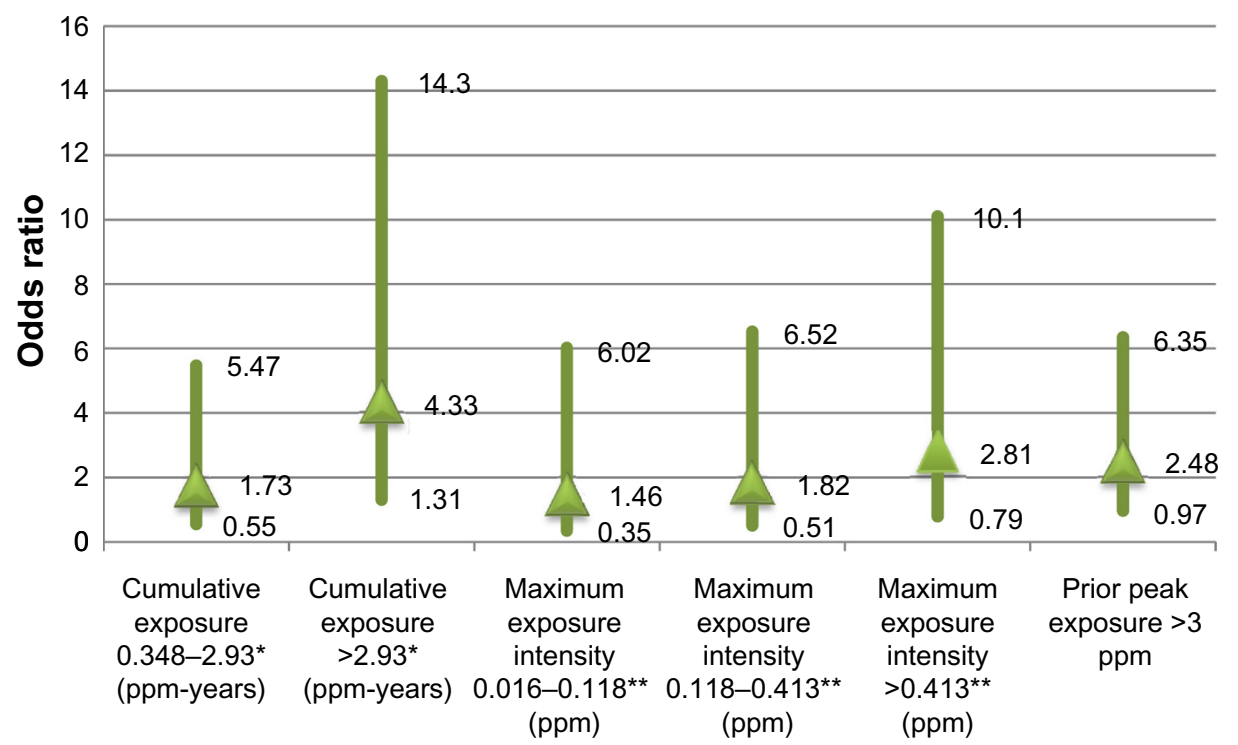

Figure I Association of MDS and benzene exposure.

Notes: Copyright () 2012, Oxford University Press. Adapted from Schnatter AR, Glass DC, Tang G, Irons RD, Rushton L. Myelodysplastic syndrome and benzene exposure among petroleum workers: an international pooled analysis. J Natl Cancer Inst. 2012;104(22):1724-1737, by permission of Oxford University Press." The upward arrows represent the odds ratio for the given study population (cases versus matched normal controls), and the lines extending from that represent the $95 \%$ confidence interval. *Reference value for odds ratio $=\mathrm{I}$ was ppm-years $<0.348$; ** reference value for odds ratio $=\mathrm{I}$ was ppm $<0.016$.

Abbreviations: MDS, myelodysplastic syndromes; ppm, parts per million.

Interestingly, autoimmune diseases such as rheumatoid arthritis, systemic lupus erythematosus, Sjögren's syndrome, or pernicious anemia, and a history of infections like pneumonia, herpes zoster, and meningitis, have been related to development of MDS. ${ }^{14}$

\section{Clonality}

Although several techniques were used in the past to determine the clonal nature of MDS cells, the issue was not completely resolved because all the techniques depended upon a skewed lyonization of the X chromosome. More recently, Walter et all used capture sequencing data and showed that up to $>90 \%$ of cells in the bone marrows of MDS samples were clonal even if the blast count was essentially zero. ${ }^{15}$ The number of clonal cells remained similar when these patients developed AML, establishing once and for all that MDS are as clonal a disease as secondary AML.

\section{Prognosis}

One of the most challenging issues in MDS has been the development of an accurate prognostic classification system. The first attempt to unify previously existing but imprecise risk-based studies was undertaken by an International MDS Risk Analysis Workshop, which performed a global analysis of seven such studies to identify critical prognostic variables. ${ }^{16}$ In what subsequently became known as the International Prognostic Scoring System (IPSS), three variables that impacted transformation to AML were identified: number of cytopenias; percentage of bone marrow (BM) blasts; and cytogenetic abnormalities, divided into good, intermediate, or poor outcome categories. This system separated MDS patients into three groups based on the hazard of $25 \%$ of patients developing leukemia into low-, intermediate-, and high-risk categories, with the intermediate-risk further stratified into intermediate-1 (Int-1) and intermediate-2 (Int-2) categories (Figure 2) ${ }^{17}$ Overall, patients with low- and Int-1-risk of transformation were collectively referred to as the lower-risk group, and those with Int-2- and high-risk as the higher-risk group. For the next 15 years, IPSS classification became the gold standard on the basis of which treatments were assigned and clinical trials were evaluated. Over the years, the system generally held well for groups of patients but was not accurately predictive for individual cases. Further refinement has been sought by the introduction of additional variables such as age, additional karyotypic subgroups, and the profundity of each individual cytopenia into the risk stratification systems by several groups and, most recently, in the publication of the revised-IPSS system. ${ }^{18}$ Where the original IPSS used published studies of 816 patients, the revised-IPSS assembled databases encompassing 7,012 patients and defined five major prognostic categories ranging from very good to very poor risk of transformation and survival. It is unlikely that any prognostic classification system devoid of comprehensive biologic and genetic information will provide the desired level of accuracy for application towards 
therapeutic decisions in individual cases. The genetics of MDS is therefore an extremely important but rapidly evolving chapter marked by discovery of novel genes and signaling pathways in rapid succession, such that the landscape has undergone a dramatic revision in just the last 3-4 years.

\section{Overview of genetic factors, genetic polymorphisms, and mutations Genetics of MDS}

Several large studies have catalogued the mutational profiles of approximately 2,000 MDS patients through high-throughput targeted genomic sequencing that shed new light on the stepwise evolution of this heterogeneous collection of syndromes and on predicting the natural history of the disease. ${ }^{19}$ Through these efforts, more than 40 genes have been identified as being recurrently mutated in MDS patients. Individual MDS patients harbor an average of fewer than a dozen mutations, some of which are the driver of the disease phenotype and others that are less consequential are termed as passenger mutations. No single driver mutation has been found to be common to all MDS patients, and neither is any mutation unique only to MDS. Genes belonging to epigenetic regulation and RNA splicing machinery are most commonly affected in MDS, with mutations of splicing factor genes alone being found in 50\% of MDS patients. The salient findings related to genetics of MDS will be reviewed here:

\section{Multifactorial pathobiology of}

\section{5q-syndrome}

Not all MDS pathology is due to mutational events as gene dosage can also play an important role in causing specific subtypes of MDS, an example being the haploinsufficiency of RPS14 leading to defective ribogenesis, which underlies the $5 \mathrm{q}$-syndrome phenotype. ${ }^{20}$ This syndrome has been recognized as a distinct clinical entity associated with prolonged survival, lower risk of transformation to leukemia in patients who present with anemia, normal or higher than normal platelets, hypolobated micromegakaryocytes in the BM, and an isolated interstitial deletion in 5q. The syndrome appears to be caused by a combined hemizygous loss of the ribosomal protein gene RPS14 and the microRNA gene mir 145 located on the commonly deleted region of 5q. Inhibition of mir 145 results in the upregulation of the transcription factor FLI1, tipping hematopoiesis in favor of megakaryocytes relative to erythrocytes resulting in anemia and an increased platelet count. ${ }^{19}$ The dysregulation of ribosomal biogenesis is not restricted to 5q-syndrome, but has recently been shown to be a more general pathogenic feature of MDS; it also links acquired anemias with congenital ones where ribogenesis is defective, such as anemia of Shwachman-Bodian Diamond syndrome, cartilage hair hypoplasia, X-linked dyskeratosis congenita, or Diamond-Blackfan anemia. ${ }^{19}$ Finally, abnormal ribogenesis also offers a molecular mechanism for the excessive apoptosis of hematopoietic cells, a hallmark of MDS biology. The steps

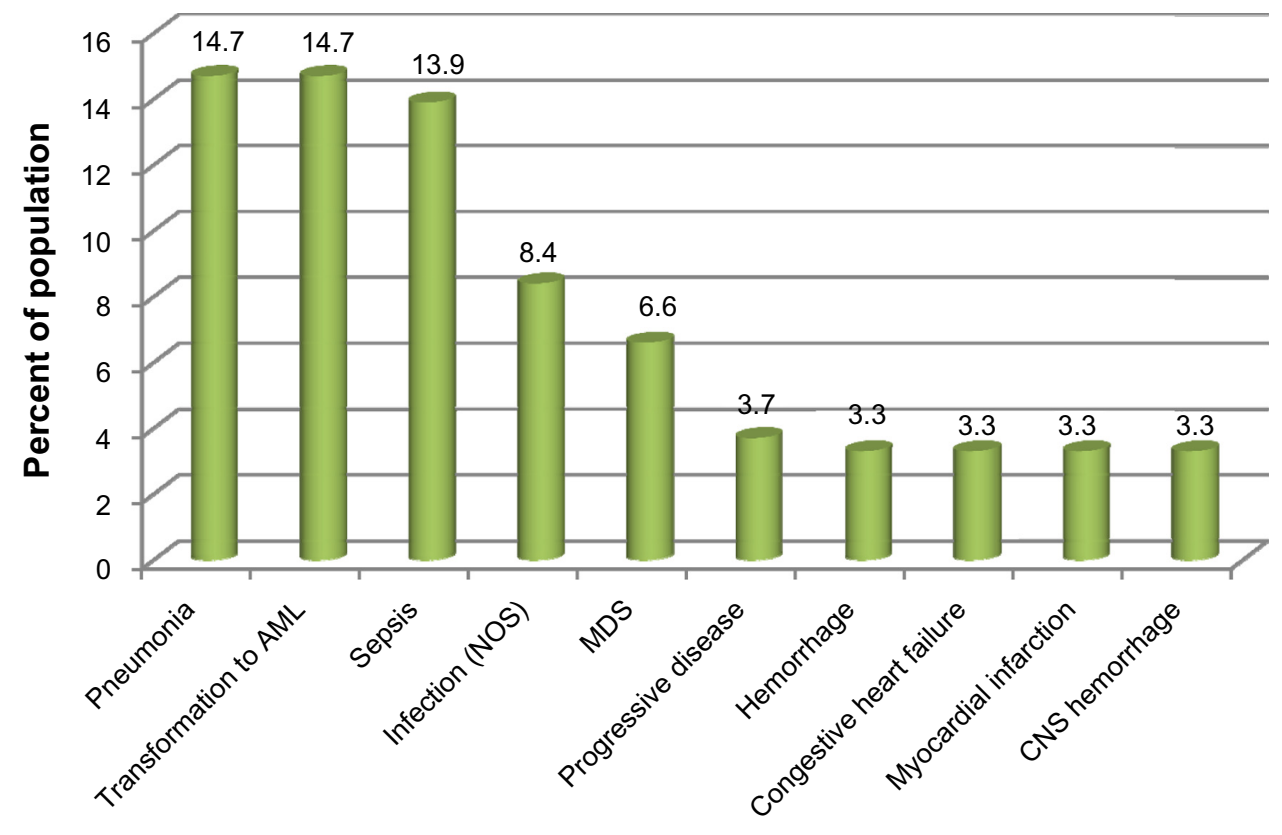

Figure 2 Ten most common causes of death in low-/Int-I-risk MDS.

Note: Copyright (C) 2010, American Cancer Society. Adapted from Dayyani F, Conley AP, Strom SS, et al. Cause of death in patients with lower-risk myelodysplastic syndrome. Cancer. 2010;1 16(9):2174-2179. ${ }^{17}$

Abbreviations: AML, acute myeloid leukemia; NOS, not otherwise specified; MDS, myelodysplastic syndromes; CNS, central nervous system. 
involved, demonstrated neatly in a mouse model, relate to stabilization of p53 as a result of Mouse Double Minute 2 Homologue (MDM2) sequestration caused by accumulation of defective ribosomal proteins. ${ }^{21}$ Because MDM2 is an E3 ligase that marks p53 for degradation, its inhibition results in continued p53 expression, cell cycle arrest, and apoptosis.

\section{Five genes of prognostic value in MDS}

Using a combination of next-generation sequencing and mass spectrometry-based genotyping, mutations were studied in 439 MDS patients and subsequently related to IPSS, specific cytopenias, the percentage of blasts, and overall survival. ${ }^{22}$ Poor survival independent of IPSS was associated with mutations in one or more of the five genes detected in $31 \%$ of MDS patients: p53, EZH2, ETV6, RUNX1, and ASXL1. These genes represent both epigenetic modifier and known oncogenes, but, while of prognostic value, provide no insight into the initiation or expansion of the MDS clone. The median survival of patients harboring any of these five mutations was closer to that of patients belonging to the next highest IPSS category, thereby providing a genetic basis for upstaging. Another important observation to emerge from this study is the confirmation of the prognostic value of p53. Among the MDS patients with a complex karyotype, presence of $p 53$ mutation was associated with significantly reduced survival, while patients with complex karyotype and no $p 53$ mutation had a natural history and median survival that was closer to that of the noncomplex cytogenetics group. Thus, the adverse prognosis of a complex karyotype can be primarily attributed to the mutational status of $p 53 .{ }^{23} \mathrm{~A}$ recent study found that $17 \%$ of patients with an isolated $\operatorname{del}(5 q)$ abnormality also harbor a $p 53$ mutation, ${ }^{24}$ while, in another study, two cases of $\operatorname{del}(5 q)$ progressing to AML were described where whole exome sequencing revealed the appearance of $p 53$ mutation only upon transformation to AML. ${ }^{25}$ Taken together, these studies suggest that mutation of p53 may be one of the molecular events necessary for leukemic transformation. For patients with a complex karyotype or isolated del(5q), sequencing for $p 53$ mutations should be performed prior to treatment in order to more accurately determine their prognosis.

\section{Epigenetic and spliceosome gene mutations}

Two studies have been published recently that used targeted sequencing of 111 and 104 genes, respectively, in 603 and 944 MDS patients, and a summary of their findings best describes the current understanding of the most commonly encountered genetic abnormalities in MDS, their general sequence of appearance, relationship to natural history of the disease, cytogenetics, and overall risk of transformation to AML. ${ }^{26,27}$ In the first study, ${ }^{26}$ a total of 738 patients were studied by targeted sequencing of 111 genes, but only 603 were MDS patients. The genes were selected on the basis of their previously demonstrated implication in MDS pathology. The first important finding of this large scale genomic-sequencing study was that a solid $22 \%$ of patients did not show any mutation in the sequenced genes. This highlights the urgent need to study the negative cases by more detailed whole exome or even whole genome sequencing. The remaining $78 \%$ of patients had one or more oncogenic mutations, most frequently affecting RNA splicing, cell signaling, DNA methylation, or chromatin modeling, thereby confirming prior observations related to these pathways being commonly dysregulated in MDS (Table 1). The median number of driver mutations was two, and it seemed that the number of mutations present in a patient had important prognostic value. On the whole, mutations were discovered in 43 genes and RNA splicing was shown to be the most commonly mutated pathway in $M D S, S F 3 B 1$ being the most frequently mutated (24\%) followed by $S R S F 2$, $U 2 A F 1$, and $Z R S R 2$. Furthermore, each mutated spliceosome gene appeared associated with a unique set of overlapping mutations, which could explain the phenotypic heterogeneity of different spliceosomal gene mutations. Mutations in TET2 (22\%) and SRSF2 (14\%) were most common after SF3B1. Mutations in $p 53, A S X L 1, S R S F 2$, and $R U N X 1$ were associated with worse survival, and allele frequency estimation showed that detection of adverse mutations even in nondominant clones negatively impacted survival. For example, $R A S$ mutations, even if present in $0.5 \%$ allele frequency, heralded an adverse prognosis. This highlights the importance of subclones. Most importantly, addition of genetic information to the IPSS scoring system improved its predictive power. Finally, a temporal order of mutational acquisition could be constructed from this study, showing that mutations in RNA splicing and DNA methylation genes were early events followed by histone posttranslational and DNA demethylation genes (TET2, ASXL1, EZH2), followed by transcription factors (RUNX1, GATA1), then $p 53$, and lastly kinase activating mutations $(N-R A S$ and $K I T)$ which were associated with late events and disease progression.

In the second study, ${ }^{27} 944$ MDS patients were screened for mutations in 104 genes using targeted deep sequencing and array-based genomic hybridization, which showed that $90 \%$ of patients had at least one mutation (median three, range $0-12$ ) even though $68 \%$ of them had normal cytogenetics. The six most commonly mutated genes found in $>10 \%$ of cases 
were similar to those found in other reports: TET2, $S F 3 B 1$, $A S X L 1, S R S F 2, D N M T 3 A$, and $R U N X 1 .^{28,29}$ In addition, $2 \%-10 \%$ of patients showed mutations involving $U 2 A F 1$, ZRSR2, STAG2, TP53, EZH2, CBL, JAK2, BCOR, IDH2, NRAS, MPL, NF1, ADM, IDH1, KRAS, PHF6, BRCC3, ETV6, and $L A M B 4$. The pathways affected by these mutations in decreasing order of frequency were: RNA splicing with mutations in as many as $64 \%$ of cases; DNA methylation; chromatin modification; transcription factors; and RAS signaling. The majority of mutations were found in higher-risk patients, but $S F 3 B 1, D N M T 3 A, J A K 2$, and $M P L$ were more common in RA/RARS patients, with $73 \%$ of cases $(133 / 183$ cases) of RA/RCMD (refractory cytopenia with multilineage dysplasia) and normal karyotype being positive for mutations. Furthermore, allele frequency revealed that the number of mutations paralleled the number of observed subpopulations. Using 14 genes combined with conventional factors yielded a novel prognostic model that separates MDS patients into four groups: low-, intermediate-, high-, and very-high-risk groups.

\section{The unique case of RARS}

In 2011, the results of the first whole exome sequencing studies in MDS patients were published simultaneously, identifying mutually exclusive novel heterogeneous point mutations in genes that code for essential proteins of the RNA-splicing machinery including SF3B1, SRSF2, U2AF1, and ZRSR2. ${ }^{28,29}$ One of the most exciting observations was the association of $S F 3 B 1$ mutations particularly with presence of ringed sideroblasts. Following exome sequencing in nine patients, target sequencing for $S F 3 B 1$ was undertaken in 2,087 patients, with 354 having MDS. ${ }^{29}$ In this study, 20\% of MDS patients showed mutation in $S F 3 B 1$ and $72 \%$ of these cases had RARS/RCMD-RS (refractory cytopenia with multilineage dysplasia and ringed sideroblasts). Mutations in SF3B1 clustered in exons $12-15$ with the hot spot resulting in the

Table I Frequency of mutations in MDS subtypes of genes involved in epigenetic and histone modifications and splicing

\begin{tabular}{|c|c|c|c|c|c|}
\hline \multirow[t]{2}{*}{ Gene } & \multicolumn{3}{|c|}{ Frequency in MDS (\%) } & \multirow[t]{2}{*}{ Prognosis } & \multirow[t]{2}{*}{ Function } \\
\hline & $\begin{array}{l}\text { RARS/RARS-T/ } \\
\text { RCMD-RS }\end{array}$ & RA/RCMD & RAEB I/2 & & \\
\hline \multicolumn{6}{|c|}{ Epigenetic and histone modifications } \\
\hline ASXLI & 4.20 & II.II & 16.81 & Poor & Plays a role in transcriptional repression of homeotic genes. \\
\hline ATRX & 1.75 & 2.22 & 1.26 & Unknown & Modulates gene expression by affecting chromatin. \\
\hline DNMTI & 1.40 & None & 0.84 & Unknown & $\begin{array}{l}\text { Maintains the methylation pattern by methylating the newly synthesized } \\
\text { DNA strand during replication. }\end{array}$ \\
\hline DNMT3A & 10.49 & 8.89 & 7.14 & Poor & Plays a role in de novo methylation. \\
\hline$E Z H 2$ & 3.15 & 4.81 & 6.72 & Poor & $\begin{array}{l}\text { Methylates histone } \mathrm{H} 3 \text {, leading to transcriptional repression of the } \\
\text { affected target gene. }\end{array}$ \\
\hline $\mathrm{IDHI}$ & 1.75 & I.II & 2.10 & Unknown & Catalyzes the oxidative decarboxylation of isocitrate to 2-oxoglutarate. \\
\hline $\mathrm{IDH} 2$ & 0.35 & 2.59 & 6.30 & Poor & Plays a role in intermediary metabolism and energy production. \\
\hline TETI & 1.75 & 6.30 & 1.68 & Unknown & $\begin{array}{l}\text { Catalyzes the conversion of methylcytosine }(5 \mathrm{mC}) \\
\text { to } 5 \text {-hydroxymethylcytosine }(\mathrm{hmC}) \text {. }\end{array}$ \\
\hline TET2 & 22.03 & 30.37 & 18.91 & Favorable & $\begin{array}{l}\text { Catalyzes the conversion of methylcytosine }(5 \mathrm{mC}) \\
\text { to } 5 \text {-hydroxymethylcytosine. }\end{array}$ \\
\hline \multicolumn{6}{|l|}{ Splicing } \\
\hline PRPF4OB & 0.70 & 0.37 & 2.10 & Unknown & Plays a role in pre-mRNA splicing. \\
\hline SFI & 0.35 & None & None & Unknown & Necessary for the ATP-dependent first step of spliceosome assembly. \\
\hline SF $3 A I$ & 0.70 & 0.37 & 0.84 & Unknown & Subunit of the splicing factor SF3A required for pre-mRNA splicing. \\
\hline SF3BI & 46.85 & 7.78 & 7.56 & Favorable & Subunit of the splicing factor SF3B required for pre-mRNA splicing. \\
\hline SFRS2 & 3.15 & II.II & 15.55 & Poor & $\begin{array}{l}\text { Subunit of an earliest ATP-dependent splicing complex required for } \\
\text { pre-mRNA splicing. }\end{array}$ \\
\hline U2AFI & 0.70 & 7.41 & 7.56 & Poor & $\begin{array}{l}\text { Plays a critical role in both constitutive and enhancer-dependent } \\
\text { splicing of pre-mRNA. }\end{array}$ \\
\hline U2AF2 & 0.70 & 0.37 & None & Unknown & $\begin{array}{l}\text { Necessary for the splicing of pre-mRNA. Binds to the polypyrimidine } \\
\text { tract of introns early during spliceosome assembly. }\end{array}$ \\
\hline ZRSR2 & None & 5.10 & 4.60 & None & Required for assembly of the prespliceosome and plays a role in splicing. \\
\hline
\end{tabular}

Note: Copyright ( $\odot$ 2012, Nature Publishing Group. Adapted from Raza A, Galili N. The genetic basis of phenotypic heterogeneity in myelodysplastic syndromes. Nat Rev Cancer. 2012; I2(12):849-859;' ' Copyright @) 2013, American Society of Hematology. Adapted from Papaemmanuil E, Gerstung M, Malcovati L, et al. Clinical and biological implications of driver mutations in myelodysplastic syndromes. Blood. 2013;122(22):3616-3627. ${ }^{26}$

Abbreviations: MDS, myelodysplastic syndrome; RA, refractory anemia; RAEBI/2, refractory anemia and excess blasts-I and -2; RARS, refractory anemia with ringed sideroblasts; RARS-T, refractory anemia with ringed sideroblasts and thrombocytosis; RCMD-RS, refractory cytopenia with multilineage dysplasia and ringed sideroblasts; ATP, adenosine triphosphate; mRNA, messenger RNA. 
amino acid change $\mathrm{K} 700 \mathrm{E}$, and all were missense mutations. RNA-sequencing data from 12 of these RARS patients showed that 94 gene sets were downregulated in patients with $S F 3 B 1$ mutation, with seven out of 50 of the most downregulated genes being related to mitochondrial function. Interestingly, among the dysregulated mitochondrial gene set, mitochondrial ribosomes were particularly affected. In other words, both cellular as well as mitochondrial ribogenesis appears defective in MDS. Furthermore, 20 genes showed differences in exon usage between $S F 3 B 1$ positive and negative cases. Clinical significance of $S F 3 B 1$ mutations $^{30}$ showed association with a higher platelet count, higher percentage of ringed sideroblasts and marrow erythroblasts, lower bone marrow blasts, and improved survival with a lower risk of transformation to AML. Mutant allelic burden estimation showed that the majority of patients had $\sim 50 \%$ of cells harboring the mutation, which is consistent with the existence of a dominant clone with the mutation in most cases and the existence of a minor mutant clone in a small fraction of patients. Finally, a study comparing $S F 3 B 1$ mutations and their clinical significance in RARS patients with thrombocytosis (RARS-T) versus patients with RARS alone found that the incidence was the same in both groups ( $85 \%$ in 33 RARS versus $87 \%$ in 111 RARS-T cases), but median survival was better for $S F 3 B 1$ mutated patients only in RARS-T cases. ${ }^{31}$ Furthermore, JAK2 mutations coexisted in $\sim 50 \%$ of RARS-T cases while no patient with RARS had a JAK2 mutation.

\section{Clinical impact of mutational heterogeneity}

An important practical question for the application of emerging genetic profiles relates to their clinical significance in determining both responsiveness to therapy and natural history of the disease. This poses a daunting challenge because all mutations are not identical; these could be missense, nonsense, frame shift, or splice site, as well as homozygous, heterozygous, hemizygous (deletion of normal allele), or compound heterozygous (each allele with a different mutation). Even within the same gene, different mutations could manifest themselves with varying phenotypes. Cataloguing of novel mutations represents the tip of the iceberg in this sense as translation into novel therapies must wait until more comprehensive maps of genetic profiles have been related to disease manifestations (Figure 3). Incorporation of genomics into therapeutic standard of care awaits, among other things, a more precise understanding of the sequence in which mutations appear, the signaling pathways they affect, the diversity of mutations in dominant versus minor subclones, and their prognostic significance.

In summary, therefore, decreasing costs of highthroughput technology has allowed for large numbers of MDS patients to be genotyped in the last few years, generating valuable information regarding the sequence of molecular events during initiation, clonal expansion, and progression of the disease, as well as more accurate prognostic predictions (Figure 4). Hopefully, development of targeted therapies will now follow in rapid succession.

\section{Development in current and emerging targeted therapies Management of anemia}

Whether patients present with lower- or higher-risk MDS, the majority present with anemia and eventually most will become RBC-transfusion dependent. Taken together, the burden of chronic anemia and repeated transfusions increases morbidity, places undue emotional and financial strains on the MDS patients, has been associated with increased risk of infections, alloimmunization, and iron overload issues, and negatively impacts the quality of life as well as compromising overall and leukemia-free survival. ${ }^{32}$ Attempts to improve anemia through use of growth factors and disease modifying agents are therefore fully justified in these patients.

\section{Hematopoietic growth factors}

Lower-risk patients including those with $\operatorname{del}(5 q)$, as well as stable higher-risk MDS patients who are anemic, should be treated with growth factors as first-line therapy. EPO and granulocyte colony stimulating factor (G-CSF) inhibit apoptosis in hematopoietic progenitor cells while promoting their growth and differentiation. Patients who are not yet transfusion dependent or who are receiving no more than 2 units of blood per month and whose serum EPO level is not high have the best chance of response to ESAs. In such patients, responses range between $60 \%$ and $70 \%$, and addition of G-CSF can rescue a subset of nonresponders to ESA alone. ${ }^{33} \mathrm{~A}$ validated model to predict response of anemic MDS patients to EPO plus G-CSF combination is defined by the Nordic studies, which assign a score for serum EPO levels $(<100,100-500$, or $>500 \mathrm{U} / \mathrm{L})$ and red cell transfusions (less or more than 2 units per month) to predict a $74 \%$ response in those scoring $>2$ points versus $7 \%$ for those scoring $<1 .{ }^{34}$ Responses generally occur within 8 weeks although some take up to 12 weeks to show benefit. The median duration of response to ESA in lower risk MDS is approximately 2 years, and $70 \%$ of relapsing patients in this group do not show disease progression but simply a loss of 


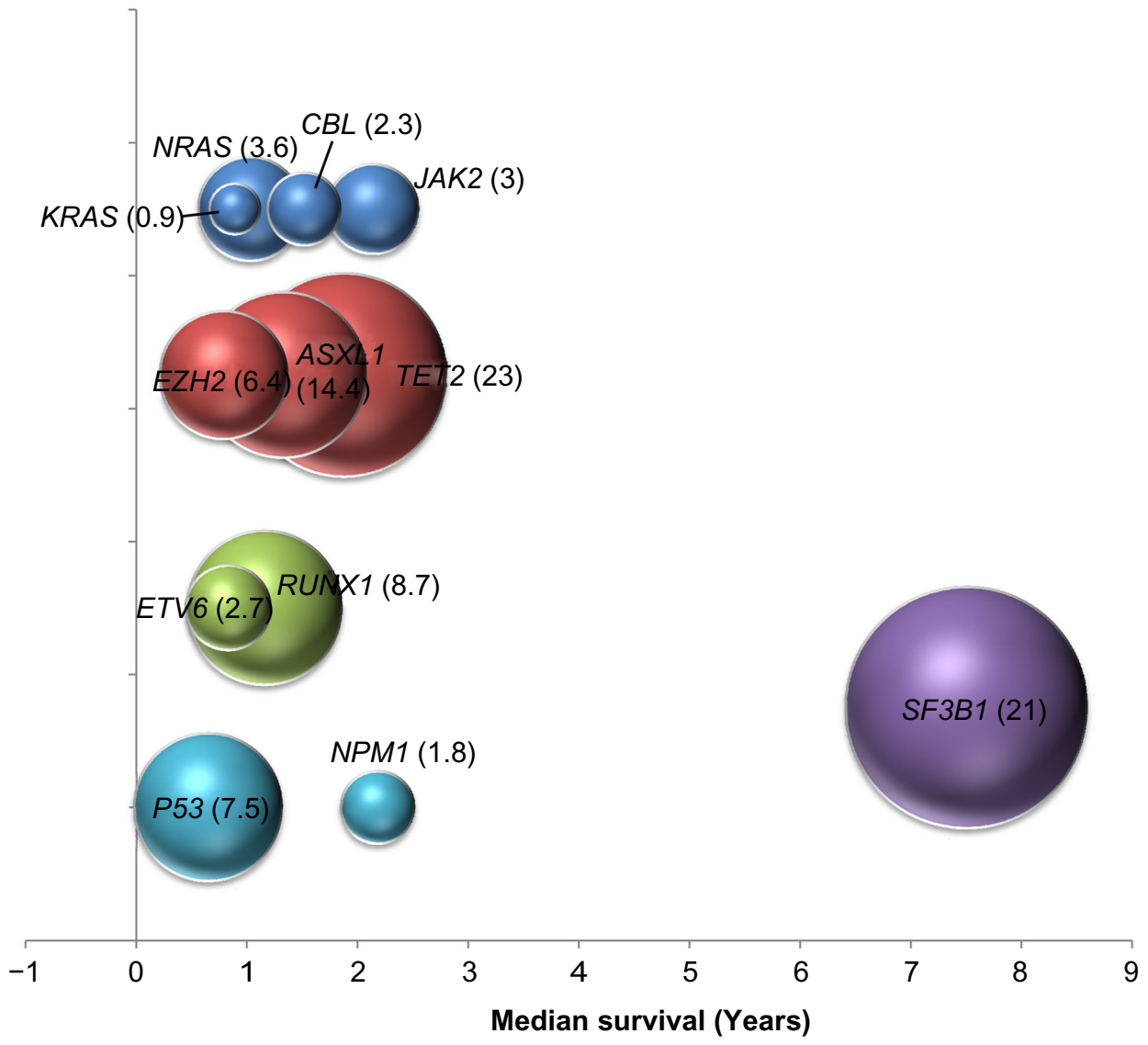

Figure 3 A bubble graph showing median survival of MDS patients with mutations in genes.

Notes: Mutations in genes involved in proliferation (blue), epigenetic regulation (red), differentiation (green), and other (blue) cellular functions relative to SF3BI gene (purple). The size of the bubble indicates the frequency of MDS patients with mutations in the indicated genes.

Abbreviation: MDS, myelodysplastic syndromes.

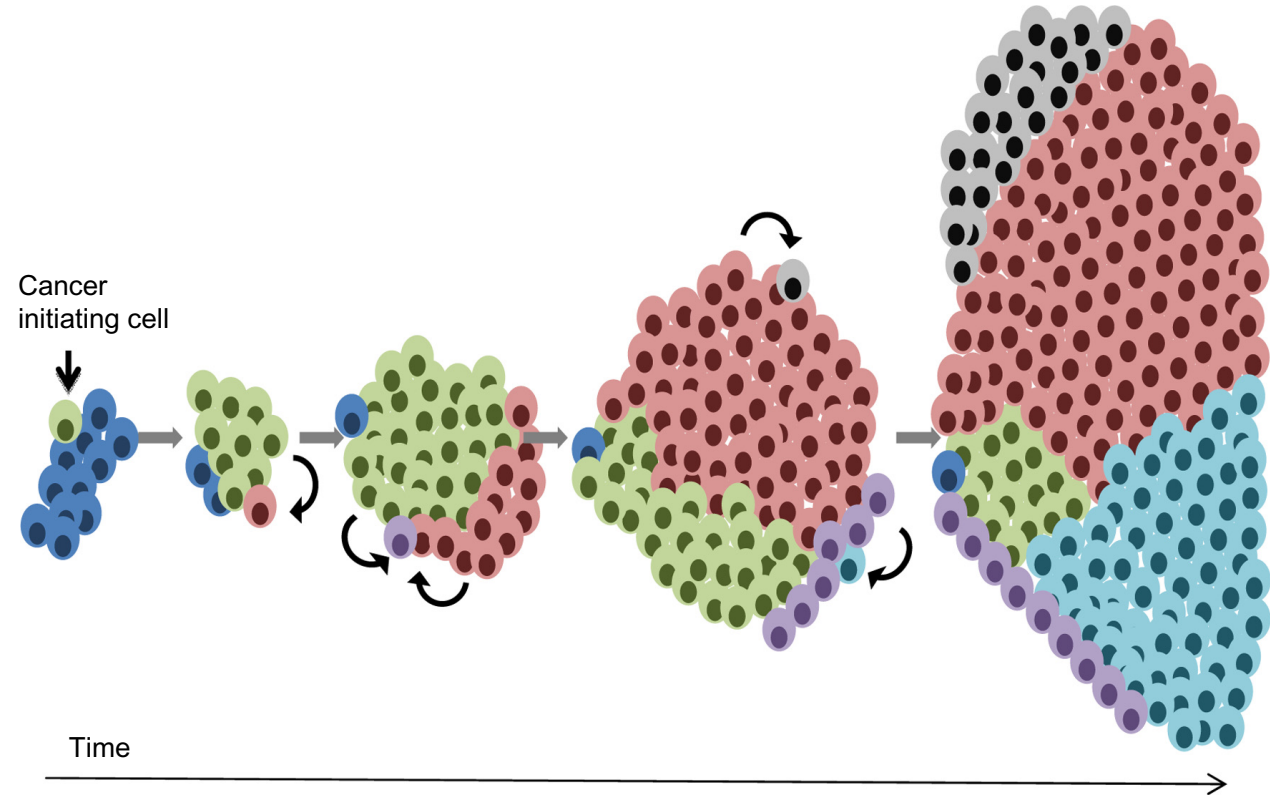

Figure 4 Clonal evolution of cancer cells in MDS.

Notes: A schematic representation showing continuous evolution of subclones from a single cell that acquired a growth advantage over its normal counterparts. This initial clone may produce distinct subclones through the course of the disease. 
response. ${ }^{35}$ Patients with lower-risk MDS and del $(5 q)$ generally have a lower response to ESAs, in the range of $\sim 40 \%$ compared to $52 \%$, and the duration of response is also shorter at a median of 1 year compared to 2 years for non-del( $5 q)$ patients. ${ }^{36}$

\section{Management of iron overload following long term RBC transfusions}

The majority of lower-risk MDS patients, many of whom survive for years, manifest disease progression, not by transforming to a higher grade of MDS, but by becoming transfusion dependent due to a loss of response to available treatments, most likely due to sequential expansion of competing clones. Some of these patients are maintained for long periods on transfusions alone. The problem is that, with each unit of blood, $250 \mathrm{mg}$ of iron is introduced into the body, with essentially no physiologic mechanism to eliminate the excess iron. With as few as ten transfusions (20 units of blood), the patient may be at risk for iron overload, although not yet experiencing signs or symptoms of this accumulation, causing end organ damage in vital organs of the body such as the heart, liver, thyroid, gonads, and the thyroid and pituitary glands. Serum ferritin level alone cannot by itself be relied upon as an accurate indicator of iron overload because it is also an acute phase reactant, but values of $>1000 \mu \mathrm{g} / \mathrm{L}$ combined with a history of transfusion of 20 units of blood are indicative of iron overload. Estimates show that, for every $500 \mu \mathrm{g} / \mathrm{L}$ increase in serum ferritin above the threshold of $1,000 \mu \mathrm{g} / \mathrm{L}$, the risk of death increases by $36 \% .{ }^{5}$ It would seem logical to attempt removal of excess iron, but a controversy exists about both the deleterious effect of iron overload as well as benefits of chelation therapy. ${ }^{37}$ Data are now emerging that suggest iron overload has a negative impact even on outcomes patients with higher-risk MDS or who have undergone stem cell transplantation, as well as the benefit of iron chelation for these individuals ${ }^{38}$ and hematologic improvement during iron chelation therapy. ${ }^{39}$ In the absence of prospective studies, chelation is presently advocated for patients with relatively good prognosis (low or Int-1 disease) or who may be candidates for transplantation. Chelation is more palatable because of the availability of oral agents and, though precise guidelines are lacking, should be considered after 20 units of blood with a consistent ferritin value in excess of $1,000 \mu \mathrm{g} / \mathrm{L}$.

\section{Treatment of anemia with disease modifying agents}

There are three US Food and Drug Administration approved agents available now. Lenalidomide is approved for transfusion-dependent anemia with lower-risk MDS and $\operatorname{del}(5 q)$ with or without additional cytogenetic abnormalities. Response to lenalidomide is seen in 55\%-65\% of such patients, with a median duration of transfusion independence between 2 and 2.5 years and best responses seen with the higher daily dose of $10 \mathrm{mg} .{ }^{40,41}$ Cytogenetic responses in these two studies ranges between $50 \%$ and $73 \%$, with $30 \%-45 \%$ complete cytogenetic responses. As noted earlier, p53 mutations are present in $\sim 20 \%$ of lower-risk MDS patients with del $(5 q)$ and are associated with a lower response to lenalidomide and a greater risk of transformation to AML. Prolonged treatment is required in responders to reduce clone size sufficiently, although discontinuation of therapy in patients with complete hematologic and cytogenetic response is associated with sustained responses for long periods of time. ${ }^{42}$ For non-del $(5 q)$ patients, after ESA failures, response to lenalidomide ranges between $25 \%$ and $30 \%,{ }^{43}$ and no effect on overall or leukemia-free survival is documented in this group so far.

While the role of hypomethylating agents is well established in higher-risk MDS patients, their place in the treatment of lower-risk patients for the management of anemia is less clear. A response rate of $30 \%-40 \%$ has been reported, but, in a trial randomizing ESA-resistant lower-risk transfusion dependent patients to azacytidine (AZA) or AZA plus EPO, transfusion independence was seen in $17 \%$ of patients with no difference between the two arms. ${ }^{44}$ No survival advantage has been recorded for these patients treated with HMAs.

Finally, immune-suppressive therapies have been used with some success. Antithymocyte globulin with or without cyclosporine can have improved cytopenias in $25 \%-40 \%$ of patients who are relatively younger patients, have short transfusion history, have $H L A-D R 15$ genotype, have presence of a PNH clone, have marrow hypocellularity, and have thrombocytopenia in addition to anemia. ${ }^{45}$

On the whole, treatment of anemia in MDS patients, except those with $\operatorname{del}(5 q)$, has generally been disappointing (being essentially palliative in nature and providing at best only temporary relief from transfusion independence) with most patients remaining either continuously or episodically dependent upon transfusions. Clearly this is an area of unmet need requiring urgent development of novel agents to relieve the elderly population from the burdens of chronic transfusions with compromised quality of life.

\section{Experimental therapies}

Various classes of agents have been tried with equivocal results for improving the anemia of MDS. A recent Phase II study of homoharringtonine, an alkaloid inhibitor of protein synthesis with activity in myeloid malignancies, found an $11 \%$ response 
rate but with a 56\% incidence of grade 3-4 myelosuppression, making this an unlikely drug for lower-risk patients. ${ }^{46}$

The cytopenias in lower-risk MDS have been shown to be due to cytokine-mediated excessive apoptosis of hematopoietic cells. ${ }^{47}$ Agents that inhibit proinflammatory cytokines such as thalidomide and lenalidomide have improved cytopenias in MDS. ARRY-614 is a dual inhibitor of p38 mitogen-activated protein kinase $\mathrm{p} 38$ and Tie2 receptor tyrosine kinase that inhibits inflammation and cytokinedependent tumor growth in preclinical models. Disruption of cytokine-mediated apoptosis in the normal progenitors and stromal cells is hypothesized to improve cytopenias in MDS and ARRY-614 can impact this step. In a Phase I dose-escalation/expansion trial of 44 evaluable patients, ARRY-614 demonstrated a 38\% multilineage hematologic improvement in patients who had failed hypomethylating agents. Responses were durable with median response duration of 5 months, with multiple patients remaining on therapy for over 12 months. ${ }^{48}$ ARRY-614 may constitute an important option for lower-risk MDS patients if these results are confirmed in larger follow-up trials.

Another agent producing encouraging multilineage responses in $\sim 30 \%$ of lower-risk MDS patients is the glutathione-S-transferase P1-1 inhibitor, ezatiostat, ${ }^{49,50}$ which causes dissociation of the enzyme from the Jun-N-terminal kinase (JNK)/c-Jun complex, leading to JNK activation by phosphorylation. Activated JNK phosphorylates c-Jun, which ultimately results in the stimulation of all hematopoietic myeloid progenitors to undergo proliferation and maturation. By analyzing a gene expression profile of pretherapy mononuclear cells using the Illumina whole genome array, it was possible to distinguish responders from nonresponders. Furthermore, genes comprising the JNK/c-Jun molecular pathway, which is known to be activated by ezatiostat, were underexpressed in patients who responded and overexpressed in patients who were nonresponders to the drug. ${ }^{51}$

Rigosertib is a multi-kinase inhibitor belonging to a class of unsaturated sulfones that exerts its effect by inhibiting polo-like kinase-1, phosphoinsitide-3 kinase, and the downstream proteins of these central pathways including cyclin D1, survivin, and c-myc. ${ }^{52}$ It differs from most kinase inhibitors in that it does not compete for the adenosine triphosphate-binding site, but instead competes for substrate binding of the kinases. An intravenous formulation in higher-risk MDS patients who failed previous treatment with hypomethylating agents showed a significant improvement in survival among responders who decreased their BM blasts by $50 \%{ }^{53} \mathrm{~A}$ Phase III trial where the endpoint is improvement in survival following HMA failure in higher-risk MDS patients has completed accrual and awaits analysis (Garcia-Manero G et al, unpublished data, 2014). The oral formulation of rigosertib has shown activity in lower-risk MDS patients, with a reported response rate of achieving complete transfusion independence in Phase I/II trials ranging between $30 \%$ and $40 \% .^{54,55} \mathrm{~A}$ genomic signature consisting of 50 hypermethylated genes associated with responders has been identified, which, if confirmed, could help preselect patients for treatment with rigosertib in the future. ${ }^{55}$

While epigenetic modifiers like AZA and decitabine are US Food and Drug Administration approved, histone deacetylase inhibitors have yielded mixed results both as single agents $^{56}$ and in combination with AZA, where vorinostat and pracinostat have yielded equivocal results so far. ${ }^{57,58}$ Other strategies have addressed the combination of HMAs with lenalidomide, mainly in higher-risk patients.

\section{Future research directions}

Despite rapid advances in understanding the genetic scaffolding of MDS in the past few years, it is a pity that our patients remain grossly underserved from these spectacular genetic revelations. Attempts to incorporate molecular and genomic information into existing systems of classification for an improved and more accurate prognostic evaluation are already under way and are likely to be validated prospectively under stringent conditions in the next few years. The desire for personalized treatment on the basis of a patient's individual genetic profile does not appear to be probable. Some of the reasons related to mutational heterogeneity (and the daunting task that lies ahead of detailed serial characterization of this mutational landscape) have already been discussed above. Another complexity, which is only now beginning to be appreciated fully, arises as a result of the heterogeneity of targets generated by the competing clones in each individual. Much like AML, MDS begins as a monoclonal disease in a single hematopoietic stem cell, which rapidly produces daughter cells that acquire a variety of mutations and, hence, signal through different pathways. There are multiple clones in each individual that are likely to gain sequential dominance in due course. Targeting one dominant clone through interrupting the pathway to which it is addicted will only produce a response for the duration of time it takes for the next clone to rear its head, addicted to a new signaling pathway for which a different drug would be required. In order to keep the disease under control, patients need to be studied longitudinally with the same rigor that is 
being applied to the one-time sequencing studies, and clinicians need to stay one step ahead of the curve by identifying the Achilles' heel of the next clone and having a targeted therapy ready for assaulting and interrupting that pathway. Only through such an approach using synthetic lethality will MDS be converted into a chronic disease that patients can live with and not die from.

\section{Conclusion}

In conclusion, MDS are malignant diseases of the elderly that are being diagnosed with increasing frequency because of the aging population. Besides the threat of leukemic transformation, anemia remains the most serious cytopenia that compromises quality of life. Correction of anemia with blood transfusions, in addition to increasing the profundity of comorbid conditions and causing iron overload with its attendant end organ damage issues, carries its own financial, emotional, and physical burden. The current therapeutic landscape is lagging behind the needs of the majority of patients. Newer disease-modifying agents can only be developed through identifying precise targets and personalizing treatments. The genomic studies currently underway have shed new light on the mutational scaffold of MDS, but have also demonstrated the patent need for more detailed and serial longitudinal genetic characterization of the disease in individual patients. Compared to the proliferation in sequencing studies, the development of new drugs is grossly lagging behind. The moment is ripe to bring the various technologic advances to the bedside for implementation of customized therapy for individual patients.

\section{Disclosure}

AR receives research funding from Onconova Therapeutics, Inc., and is a speaker for Novartis. The authors report no other conflicts of interest in this work.

\section{References}

1. Cogle CR, Craig BM, Rollison DE, List AF. Incidence of the myelodysplastic syndromes using a novel claims-based algorithm: high number of uncaptured cases by cancer registries. Blood. 2011;117(26): 7121-7125.

2. de Swart L, Smith A, Fenaux P, et al. Early mortality in 1000 newly diagnosed MDS patients with low- and intermediate-1 risk MDS in the European Leukemianet MDS (EUMDS) Registry. Blood. 2012;120(21):3830.

3. Heptinstall KV. P124 Quality of life (QoL) in myelodysplastic syndromes (MDS): an update of results from US and European patient forums. Leuk Res. 2007;31(Suppl 1):S107.

4. Goldberg SL, Chen E, Corral M, et al. Incidence and clinical complications of myelodysplastic syndromes among United States Medicare beneficiaries. J Clin Oncol. 2010;28(17):2847-2852.

5. Malcovati L, Porta MG, Pascutto C, et al. Prognostic factors and life expectancy in myelodysplastic syndromes classified according to WHO criteria: a basis for clinical decision making. J Clin Oncol. 2005;23(30):7594-7603.
6. Malcovati L, Germing U, Kuendgen A, et al. Time-dependent prognostic scoring system for predicting survival and leukemic evolution in myelodysplastic syndromes. J Clin Oncol. 2007;25(23):3503-3510.

7. Ma X, Does M, Raza A, Mayne ST. Myelodysplastic syndromes: incidence and survival in the United States. Cancer. 2007;109(8): 1536-1542.

8. Sekeres MA. Epidemiology, natural history, and practice patterns of patients with myelodysplastic syndromes in 2010. J Natl Compr Canc Netw. 2011;9(1):57-63.

9. Kuendgen A, Matsuda A, Germing U. Differences in epidemiology of MDS between Western and Eastern countries: Ethnic differences or environmental influence? Leuk Res. 2007;31(1):103-104.

10. Sekeres MA, Schoonen WM, Kantarjian H, et al. Characteristics of US patients with myelodysplastic syndromes: results of six cross-sectional physician surveys. J Natl Cancer Inst. 2008;100(21):1542-1551.

11. Schnatter AR, Glass DC, Tang G, Irons RD, Rushton L. Myelodysplastic syndrome and benzene exposure among petroleum workers: an international pooled analysis. J Natl Cancer Inst. 2012;104(22): 1724-1737.

12. Honda Y, Delzell E, Cole P. An updated study of mortality among workers at a petroleum manufacturing plant. J Occup Environ Med. 1995;37(2):194-200.

13. Ma W, Kantarjian H, Zhang K, et al. Significant association between polymorphism of the erythropoietin gene promoter and myelodysplastic syndrome. BMC Med Genet. 2010;11:163.

14. Kristinsson SY, Bjorkholm M, Hultcrantz M, Derolf AR, Landgren O, Goldin LR. Chronic immune stimulation might act as a trigger for the development of acute myeloid leukemia or myelodysplastic syndromes. J Clin Oncol. 2011;29(21):2897-2903.

15. Walter MJ, Shen D, Ding L, et al. Clonal architecture of secondary acute myeloid leukemia. N Engl J Med. 2012;366(12):1090-1098.

16. Greenberg P, Cox C, LeBeau MM, et al. International scoring system for evaluating prognosis in myelodysplastic syndromes. Blood. 1997;89(6):2079-2088.

17. Dayyani F, Conley AP, Strom SS, et al. Cause of death in patients with lower-risk myelodysplastic syndrome. Cancer. 2010;116(9): 2174-2179.

18. Greenberg PL, Tuechler H, Schanz J, et al. Revised international prognostic scoring system for myelodysplastic syndromes. Blood. 2012;120(12):2454-2465.

19. Raza A, Galili N. The genetic basis of phenotypic heterogeneity in myelodysplastic syndromes. Nat Rev Cancer. 2012;12(12):849-859.

20. Ebert BL, Pretz J, Bosco J, et al. Identification of RPS14 as a 5q- syndrome gene by RNA interference screen. Nature. 2008;451(7176):335-339.

21. Barlow JL, Drynan LF, Hewett DR, et al. A p53-dependent mechanism underlies macrocytic anemia in a mouse model of human $5 \mathrm{q}$ - syndrome. Nat Med. 2010;16(1):59-66.

22. Bejar R, Stevenson K, Abdel-Wahab O, et al. Clinical effect of point mutations in myelodysplastic syndromes. $N$ Engl J Med. 2011;364(26):2496-2506.

23. Lindsley RC, Ebert BL. Molecular pathophysiology of myelodysplastic syndromes. Annu Rev Pathol. 2013;8:21-47.

24. Sebaa A, Ades L, Baran-Marzack F, et al. Incidence of 17p deletions and TP53 mutation in myelodysplastic syndrome and acute myeloid leukemia with 5q deletion. Genes Chromosomes Cancer. 2012;51(12): 1086-1092.

25. Pellagatti A, Fernandez-Mercado M, Di Genua C, et al. Whole-exome sequencing in $\operatorname{del}(5 q)$ myelodysplastic syndromes in transformation to acute myeloid leukemia. Leukemia. Epub December 24, 2013.

26. Papaemmanuil E, Gerstung M, Malcovati L, et al. Clinical and biological implications of driver mutations in myelodysplastic syndromes. Blood. 2013;122(22):3616-3627; quiz 3699.

27. Haferlach T, Nagata Y, Grossmann V, et al. Landscape of genetic lesions in 944 patients with myelodysplastic syndromes. Leukemia. 2014;28(2):241-247.

28. Yoshida K, Sanada M, Shiraishi Y, et al. Frequent pathway mutations of splicing machinery in myelodysplasia. Nature. 2011;478(7367): 64-69. 
29. Papaemmanuil E, Cazzola M, Boultwood J, et al. Somatic SF3B1 mutation in myelodysplasia with ring sideroblasts. $N$ Engl J Med. 2011;365(15):1384-1395.

30. Malcovati L, Papaemmanuil E, Bowen DT, et al. Clinical significance of SF3B1 mutations in myelodysplastic syndromes and myelodysplastic/ myeloproliferative neoplasms. Blood. 2011;118(24):6239-6246.

31. Broseus J, Alpermann T, Wulfert M, et al. Age, JAK2(V617F) and SF3B1 mutations are the main predicting factors for survival in refractory anaemia with ring sideroblasts and marked thrombocytosis. Leukemia. 2013;27(9):1826-1831.

32. Platzbecker U, Hofbauer LC, Ehninger G, Holig K. The clinical, quality of life, and economic consequences of chronic anemia and transfusion support in patients with myelodysplastic syndromes. Leuk Res. 2012; 36(5):525-536.

33. Hellstrom-Lindberg E, van de Loosdrecht A. Erythropoiesis stimulating agents and other growth factors in low-risk MDS. Best Pract Res Clin Haematol. 2013;26(4):401-410.

34. Hellstrom-Lindberg E, Gulbrandsen N, Lindberg G, et al. A validated decision model for treating the anaemia of myelodysplastic syndromes with erythropoietin + granulocyte colony-stimulating factor: significant effects on quality of life. Br J Haematol. 2003;120(6):1037-1046.

35. Park S, Grabar S, Kelaidi C, et al. Predictive factors of response and survival in myelodysplastic syndrome treated with erythropoietin and G-CSF: the GFM experience. Blood. 2008;111(2):574-582.

36. Kelaidi C, Park S, Brechignac S, et al. Treatment of myelodysplastic syndromes with $5 \mathrm{q}$ deletion before the lenalidomide era; the GFM experience with EPO and thalidomide. Leuk Res. 2008;32(7): 1049-1053.

37. Steensma DP. The relevance of iron overload and the appropriateness of iron chelation therapy for patients with myelodysplastic syndromes: a dialogue and debate. Curr Hematol Malig Rep. 2011;6(2):136-144.

38. Leitch HA. Controversies surrounding iron chelation therapy for MDS. Blood Rev. 2011;25(1):17-31.

39. Molteni A, Riva M, Pellizzari A, et al. Hematological improvement during iron-chelation therapy in myelodysplastic syndromes: the experience of the "Rete Ematologica Lombarda". Leuk Res. 2013;37(10): 1233-1240.

40. List A, Dewald G, Bennett J, et al. Lenalidomide in the myelodysplastic syndrome with chromosome 5q deletion. NEngl J Med. 2006;355(14): 1456-1465.

41. Fenaux P, Giagounidis A, Selleslag D, et al. A randomized phase 3 study of lenalidomide versus placebo in RBC transfusion-dependent patients with Low-/Intermediate-1-risk myelodysplastic syndromes with del5q. Blood. 2011;118(14):3765-3776.

42. Giagounidis AA, Kulasekararaj A, Germing U, et al. Long-term transfusion independence in $\operatorname{del}(5 q)$ MDS patients who discontinue lenalidomide. Leukemia. 2012;26(4):855-858.

43. Raza A, Reeves JA, Feldman EJ, et al. Phase 2 study of lenalidomide in transfusion-dependent, low-risk, and intermediate-1 risk myelodysplastic syndromes with karyotypes other than deletion 5q. Blood. 2008;111(1):86-93.

44. Boehrer S, Beyne-Rauzy O, Prebet T, et al. Interim results of a randomized phase II trial of azacitidine (AZA) +/- epo in lower risk myelodysplastic syndrome (MDS) resistant to an erythropoietic stimulating agent (ESA) alone. Blood. 2010;116(21):1880.
45. Fenaux P, Ades L. How we treat lower-risk myelodysplastic syndromes. Blood. 2013;121(21):4280-4286.

46. Daver N, Vega-Ruiz A, Kantarjian HM, et al. A phase II open-label study of the intravenous administration of homoharringtonine in the treatment of myelodysplastic syndrome. Eur J Cancer Care (Engl). 2013;22(5):605-611.

47. Raza A, Gezer S, Mundle S, et al. Apoptosis in bone marrow biopsy samples involving stromal and hematopoietic cells in 50 patients with myelodysplastic syndromes. Blood. 1995;86(1):268-276.

48. Komrokji RS, List AF, Khoury HJ, et al. Phase 1 dose-escalation/expansion study of the p38/Tie2 inhibitor ARRY-614 in patients with IPSS low/Int-1 risk myelodysplastic syndromes. Blood. 2011;118(21):118.

49. Raza A, Galili N, Smith SE, et al. A phase 2 randomized multicenter study of 2 extended dosing schedules of oral ezatiostat in low to intermediate-1 risk myelodysplastic syndrome. Cancer. 2012;118(8): 2138-2147.

50. Raza A, Galili N, Mulford D, et al. Phase 1 dose-ranging study of ezatiostat hydrochloride in combination with lenalidomide in patients with non-deletion (5q) low to intermediate-1 risk myelodysplastic syndrome (MDS). J Hematol Oncol. 2012;5:18.

51. Galili N, Tamayo P, Botvinnik OB, et al. Prediction of response to therapy with ezatiostat in lower risk myelodysplastic syndrome. $J$ Hematol Oncol. 2012;5:20.

52. Reddy MV, Venkatapuram P, Mallireddigari MR, et al. Discovery of a clinical stage multi-kinase inhibitor sodium (E)-2-\{2-methoxy-5$\left[\left(2^{\prime}, 4^{\prime}, 6^{\prime}\right.\right.$-trimethoxystyrylsulfonyl)methyl]phenylamino $\}$ acetate $(\mathrm{ON}$ 01910.Na): synthesis, structure-activity relationship, and biological activity. J Med Chem. 2011;54(18):6254-6276.

53. Silverman LR, Raza A, Sloand EM, Greenberg PL, Wilhelm FE. Overall Survival In Myelodysplastic Syndrome or Acute Myeloid Leukemia Patients Treated with On 01910.Na Correlates with Bone Marrow Blast Response. Blood. 2010;116(21):3998.

54. Komrokji RS, Raza A, Lancet JE, et al. Phase I clinical trial of oral rigosertib in patients with myelodysplastic syndromes. Br J Haematol. 2013;162(4):517-524

55. Raza A, Tycko B, Lee S, et al. Oral rigosertib (ON 01910.Na) treatment produces an encouraging rate of transfusion independence in lower risk myelodysplastic syndromes (MDS) patients; a genomic methylation profile is associated with responses. 55th American Society of Hematology (ASH) Annual Meeting and Exposition; Blood. 2013;122(21):2745.

56. Flinn I, Lang E, Raefsky E, et al. Preliminary Results of a Phase II Trial of Panobinostat (LBH589) In Refractory Myelodysplastic Syndromes (MDS) Patients. Blood. 2010;116(21):1636-1637.

57. Silverman LR, Verma A, Odchimar-Reissig R, et al. A phase I/II study of vorinostat, an oral histone deacetylase inhibitor, in combination with azacitidine in patients with the myelodysplastic syndrome (MDS) and acute myeloid leukemia (AML). Initial results of the phase I trial: A New York Cancer Consortium. 44th American Society of Clinical Oncology (ASCO) Annual Meeting; J Clin Oncol. 2008;26(15S):7000.

58. Quintás-Cardama A, Kantarjian HM, Ravandi F, et al. Very High Rates of Clinical and Cytogenetic Response with the Combination of the Histone Deacetylase Inhibitor Pracinostat (SB939) and 5-Azacitidine in High-Risk Myelodysplastic Syndrome. Blood. 2012;120(21):3821.
Advances in Genomics and Genetics

\section{Publish your work in this journal}

Advances in Genomics and Genetics is an international, peer reviewed, open access journal that focuses on new developments in characterizing the human and animal genome and specific gene expressions in health and disease. Particular emphasis will be given to those studies that elucidate genes, biomarkers and targets in the development of new or improved therapeutic

\section{Dovepress}

interventions. The journal is characterized by the rapid reporting of reviews, original research, methodologies, technologies and analytics in this subject area. The manuscript management system is completely online and includes a very quick and fair peer-review system. Visit http://www.dovepress.com/ testimonials.php to read real quotes from published authors. 